\title{
Percepciones y acciones de las y los estudiantes de la Universidad Autónoma del Estado de México respecto a la educación en el desarrollo sostenible
}

\author{
Perceptions and actions of Autonomous University of the State of Mexico \\ students in regard to a sustainable development education
}

\section{Percepções e ações dos alunos da Universidade Autônoma do Estado do México em relação à educação em desenvolvimento sustentável}

Jenny García Guerrero

Universidad Autónoma del Estado de México, México

Ips.jennyfer.07@hotmail.com https://orcid.org/0000-0002-8049-7090

Cristina María Guadalupe García Rendón Arteaga Universidad Autónoma del Estado de México, México

cmggra@gmail.com https://orcid.org/0000-0002-7384-4189

\section{Resumen}

Hoy en día la relación entre la naturaleza y la sociedad se encuentra en un estado crítico. Las grandes industrias han transgredido los tiempos del mundo natural, rompiendo el equilibrio de los ecosistemas: un daño irreversible al planeta. Al mismo tiempo, las personas, en algunas ciudades, han cambiado el sentido de lo que se necesita para vivir. La perspectiva de la calidad de vida se enfoca en la acumulación de bienes, se piensa que más es mejor, lo que provoca un consumismo sin importar las repercusiones a nivel personal, social y ambiental. Ante este panorama, se consideró importante conocer la relación que mantienen los estudiantes de la Universidad Autónoma del Estado de México (UAEMéx) con el desarrollo sostenible. Por ello, se realizó una investigación cuyo objetivo fue identificar y analizar las percepciones y acciones relacionadas con el modo de vida sostenible o no sostenible en este grupo en particular. La 


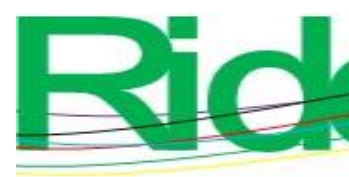

Revista Iberoamericana para la

Investigación y el Desarrollo Educativo

ISSN 2007 - 7467

investigación fue de tipo cualitativo y de nivel descriptivo; se basó principalmente en dos categorías: 1) la construcción del ethos sostenible y 2) la educación sostenible en los estudiantes de la UAEMex, Campus Toluca. En la primera, se profundiza en las percepciones y acciones que realizan los universitarios respecto a la sostenibilidad; y en la segunda, se inquirió sobre su perspectiva con relación al tema. El estudio se realizó a partir de una muestra intencional de 47 alumnos de tres áreas del conocimiento: ciencias de la salud (Medicina y Enfermería), ingeniería y tecnología (Ingeniería y Arquitectura) y ciencias de la educación y humanidades (Psicología); a esta muestra se le realizó una entrevista semiestructurada.

De entre los resultados de la investigación, la cual se realizó en el año 2017, destaca que los estudiantes reconocen que la crisis ambiental es causada por los seres humanos, por lo tanto, su resolución y los cuidados ambientales deben ser responsabilidad de estos. Además, siguiendo con los resultados, el universitario sí lleva a cabo acciones relacionadas con el desarrollo sostenible, que provienen de hábitos y costumbres adquiridos principalmente en la familia. Sin embargo, la percepción que tienen es que en la universidad no se cuentan con las condiciones suficientes para llevar a cabo acciones en pro del ambiente. En esa línea, las propuestas que dan para mejorar el desarrollo sostenible en la universidad son hacer mayor difusión de la información relacionada al cuidado del ambiente, contar con contenedores para material de reciclaje y desechos y realizar actividades relacionadas con el cuidado del ambiente en las que se involucre a la comunidad estudiantil.

La información de esta investigación permitirá realizar propuestas en el campo educativo a nivel licenciatura en la UAEMéx. Es necesario poner énfasis en una educación formal e informal para el desarrollo sostenible, que permita a los habitantes del planeta solventar las necesidades actuales, sin comprometer las necesidades futuras, con responsabilidad y solidaridad.

Palabras clave: educación, ética, sostenibilidad. 


\section{Abstract}

Nowadays, the relation between nature and society is in critical condition. The big industries have transgressed the times of this relation, breaking the equilibrium of the ecosystems. This is provoking an irreversible damage to our planet. Simultaneously, people in some cities have changed the sense of what is needed to live. The perspective of the quality of life focusses on the accumulation of goods and it is thought that more is better, causing a consumerism no matter the repercussions at a personal, social and environmental level. In light of this situation, it was considered important to know the relation in which the students of the UAEM are with the sustainable development. For this reason, it was made an investigation whose goal was to identify and analyze the perceptions and actions related to the sustainable or unsustainable lifestyle in the students of the UAEM. The investigation was qualitative and descriptive. It was based mainly on two categories: the building of sustainable ethos and the sustainable education in students of the UAEM in Toluca. The first category go far into the perceptions and actions that the university students execute regarding to the sustainability. In the second one, it was known the perspective of the students regarding to the topic. The study was made from a purposive sample of 47 students from three different areas of knowledge: Health Sciences (Medicine and Nursing), Engineering and Technology (Engineering and Architecture) and Learning Sciences and Arts (Psychology) in Toluca, and they answered to a semi-structured interview.

From the results of the investigation made at the UAEM in the year 2017, it is highlighted: The students acknowledge that the environmental crisis is caused by the human being, therefore, the recuperation of the environment and its citizens is responsibility of the human being; the university student in fact execute actions related to the sustainable development, and that actions come from habits and customs acquired mainly in the family; the perception that the students have is that the University does not have the conditions to accomplish actions in the interest of the environment ; the proposals that the students gave in order to improve the sustainable development at the University are: give wider diffusion of the information regarding to the care of environment, to have containers for recycling material and wastes, and conduct activities related to the care of environment in which the students can be involved. 

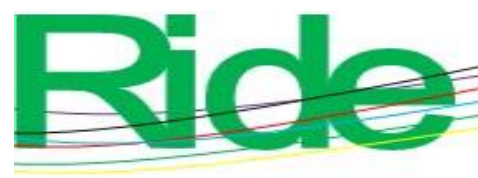

Revista Iberoamericana para la

Investigación y el Desarrollo Educativo

ISSN $2007-7467$

The information of the investigation will allow the development of proposals in the education sector at a bachelor level at the UAEM. Then it becomes necessary to emphasize a formal and informal education for the sustainable development that allows the inhabitants of the world address the current necessities, without compromising the future necessities, with responsibility and solidarity.

Keywords: education, ethics, sustainability.

\section{Resumo}

Atualmente, a relação entre natureza e sociedade está em estado crítico. As grandes indústrias transgrediram os tempos do mundo natural, quebrando o equilíbrio dos ecossistemas: um dano irreversível ao planeta. Ao mesmo tempo, as pessoas, em algumas cidades, mudaram o significado do que é necessário para viver. A perspectiva de qualidade de vida centra-se na acumulação de bens, pensa-se que mais é melhor, o que provoca um consumismo independentemente das repercussões a nível pessoal, social e ambiental. Diante desse panorama, considerou-se importante conhecer a relação que os alunos da Universidade Autônoma do Estado do México (UAEMéx) mantêm com o desenvolvimento sustentável. Para tanto, foi realizada uma pesquisa cujo objetivo foi identificar e analisar as percepções e ações relacionadas ao modo de vida sustentável ou insustentável nesse grupo em particular. A pesquisa foi de nível qualitativo e descritivo; Baseava-se principalmente em duas categorias: 1) construção de ethos sustentável e 2) educação sustentável para estudantes da UAEMex, Campus de Toluca. Na primeira, aprofunda as percepções e ações que os universitários realizam em relação à sustentabilidade; e no segundo, indagou sobre sua perspectiva em relação ao assunto. O estudo foi realizado a partir de uma amostra intencional de 47 alunos de três áreas do conhecimento: ciências da saúde (Medicina e Enfermagem), engenharia e tecnologia (Engenharia e Arquitetura) e ciências da educação e humanas (Psicologia); Esta amostra foi submetida a uma entrevista semiestruturada.

Entre os resultados da pesquisa, que foi realizada no ano de 2017, destaca que os estudantes reconhecem que a crise ambiental é causada por seres humanos, portanto, sua resolução e cuidado ambiental devem ser de sua responsabilidade. Além disso, acompanhando os resultados, 

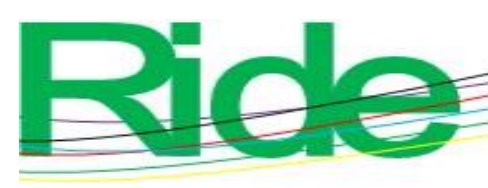

Revista Iberoamericana para la Investigación y el Desarrollo Educativo ISSN $2007-7467$

a universidade realiza ações relacionadas ao desenvolvimento sustentável, provenientes de hábitos e costumes adquiridos principalmente na família. No entanto, a percepção que eles têm é que a universidade não tem condições suficientes para realizar ações em favor do meio ambiente. Nesta linha, as propostas que dão para melhorar o desenvolvimento sustentável na universidade são fazer maior difusão da informação relacionada ao cuidado do ambiente, contar com recipientes para material de reciclagem e desperdício e realizar atividades relacionadas ao cuidado do ambiente em qual a comunidade estudantil está envolvida.

As informações desta investigação permitirão fazer propostas no campo educacional em nível universitário na UAEMéx. É necessário enfatizar a educação formal e informal para o desenvolvimento sustentável, que permita aos habitantes do planeta satisfazer as necessidades atuais, sem comprometer as necessidades futuras, com responsabilidade e solidariedade.

Palavras-chave: educação, ética, sustentabilidade.

\section{La educación para el desarrollo sostenible}

\section{Punto de partida, la problemática actual}

Desde el origen de los tiempos, el ser humano ha estado en contacto con un ambiente que le ha proporcionado los medios suficientes para poder vivir; ha aprovechado el medio a su alrededor para satisfacer sus necesidades básicas. Sin embargo, con el paso de los años, el planeta se fue poblando de más humanos que ya no solo pensaban en sus necesidades básicas, sino en satisfacer necesidades creadas culturalmente. En consecuencia, se le empezó a exigir al medio ambiente más de lo que este podía brindar.

De igual manera, las personas y sociedades fueron perdiendo la conciencia de cuidar aquello que les había permitido vivir, pusieron de lado las enseñanzas inculcadas por sus ancestros respecto al cuidado del entorno y dejaron en el descuido al resto de los seres vivos, incluida la madre tierra, llegando, en algunos casos, al punto de la extinción. 

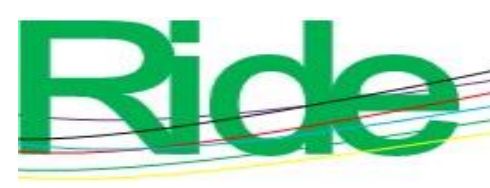

Revista Iberoamericana para la Investigación y el Desarrollo Educativo ISSN $2007-7467$

Posteriormente, pensando en el bienestar de la sociedad, se promovió el desarrollo industrial sin tomar en cuenta los riesgos ecológicos que provocaría dicha promoción. Se encontraban enfocados en lograr un desarrollo que permitiera que las comunidades tuvieran mayor crecimiento y, por lo tanto, mejor calidad de vida. Pero ese desarrollo, de un tiempo para acá, se ha convertido en un desarrollismo, "hemos desarrollado una cultura en la que 'más' parece ser siempre 'mejor"' (Novo, 2010, p. 62).

El desarrollismo ha provocado en los seres humanos una falsa necesidad de poseer bienes que la misma sociedad ha hecho creer que son indispensables, impulsando un consumo desmedido que a la vez ha provocado un crecimiento descontrolado en las sociedades modernas; esto, con el paso del tiempo, ha traído daños no menores al ecosistema. Aunado a todo ello, existen personas que tienen la creencia que el recurso de la naturaleza es inagotable:

Hemos vivido (y todavía vivimos en parte) en una especie de espejismo en el que se piensa que la razón puede dar cuenta de la vida dejando de lado la imaginación, los sentimientos o los valores. Igualmente se sigue funcionando como si los bienes de la Naturaleza fuesen ilimitados (Novo, 2003, pp. 6-7).

Asimismo, el desarrollismo también ha contribuido al aumento de la economía de manera desproporcionada: solo ha beneficiado a un grupo reducido de personas, lo que, consecuentemente, ha traído una división entren los países ricos del norte y los países pobres del sur; a pesar de que aquellos son abastecidos por estos, no hay equidad de por medio.

Una de las explicaciones para entender la crisis entre el ser humano y el ambiente refiere a la confusión entre las necesidades y los deseos que se generan en las personas de las sociedades modernas. Al respecto, Parellada (2008) comenta que "no todos los deseos de los individuos se deben considerar necesidades, por muy fuerte que sea el reclamo del deseo o por muy intensa que sea la frustración experimentada por el individuo cuando no se satisface” (p. 78).

Otra es la modificación de los valores prioritarios para la sociedad, que a la vez se han reflejado en las acciones de las personas, lo que ha provocado que se pase por encima de los tiempos de la naturaleza. 


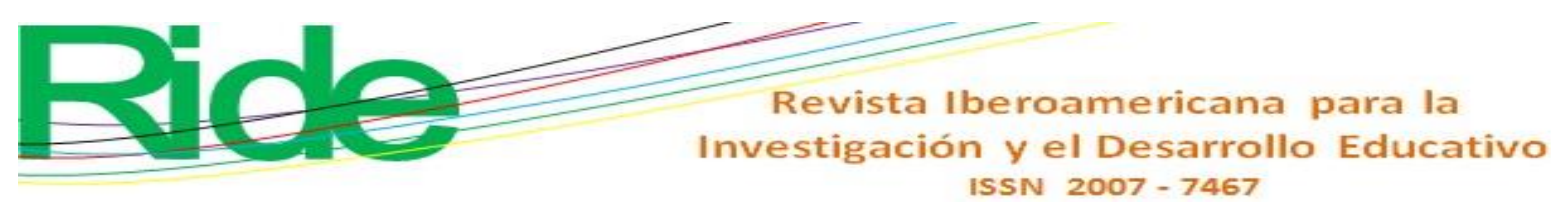

La crisis ambiental a la que nos enfrentamos actualmente como civilización no tiene que ver solamente con estilos de desarrollo y formas de crecimiento económico o con el incremento desmedido y acelerado de la población, la tecnología y sus productos contaminantes, así como, la sobreexplotación de los recursos naturales, sino también por la pérdida de valores y la falta de certeza en el futuro, panorama que representa la necesidad de buscar nuevos caminos y el replanteamiento de valores y estilos de vida (García, 2011, p. 88).

Por otra parte, al realizar un acercamiento a la esfera de las universidades, estas instituciones educativas también han aumentado sus actividades que generan contaminantes y dañan al ambiente; el gran número de habitantes que acogen, a causa del crecimiento de la población, es una de las razones. En ese sentido, Juárez, Juan y Estrada (2016) mencionan que "la universidad genera impactos en el medio ambiente, principalmente, los asociados con la generación de residuos, transporte vehicular, descarga de aguas residuales y emisión de sustancias contaminantes a la atmosfera. La universidad tiene una huella ecológica" (p. 2).

A estas alturas, como lo menciona Novo, "lo que está en peligro no es solamente la calidad de vida, sino la propia vida" (2012, p. 12).

\section{El desarrollo sostenible}

Lo anterior ha dado lugar a una serie de preguntas acerca de cuáles son los caminos más viables para la recuperación de nuestro ecosistema, o, dicho de otra manera, cuál es la manera más apropiada para dañar lo menos posible nuestro entorno. Una de las respuestas conduce a propiciar el desarrollo sostenible.

El desarrollo sostenible es "aquel que satisface las necesidades de las generaciones presentes sin comprometer la capacidad de las futuras generaciones para satisfacer las propias" (Novo, 2009b, p. 208). En otras palabras, lo que se está buscando hoy en día son alternativas viables para solventar las necesidades, pero de tal forma que se dañe lo menos posible al planeta y sin poner en riesgo la satisfacción de las necesidades básicas de las generaciones futuras. 


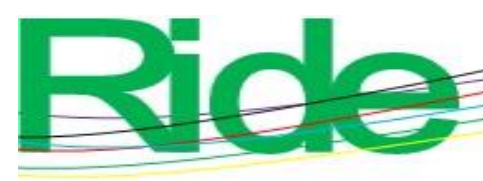

Revista Iberoamericana para la Investigación y el Desarrollo Educativo ISSN $2007-7467$

El futuro sustentable se construye a través de una epistemología política y de una ética de la responsabilidad hacia la vida; ello implica abrir el campo de lo posible dentro de las condiciones cósmicas, geofísicas y ecológicas del planeta vivo que habitamos, y de las condiciones humanas para pensar y conducir a través del conocimiento, del saber, del sentido, del diálogo, de la responsabilidad ética y de la acción política, las posibles formas sustentables de apropiación y transformación de la naturaleza. (Leff, 2011, p. 96)

Actuar con responsabilidad es la clave de la sostenibilidad: pensar que las generaciones actuales, como habitantes del planeta, tienen un compromiso con las nuevas generaciones, las cuales evidentemente necesitarán de los recursos que proporciona la naturaleza. Por lo que es necesario dejar de lado el egoísmo y empezar a actuar a favor de la regeneración de nuestro planeta de forma responsable. Esto no es imposible, hay esfuerzos comunitarios que lo están llevando a cabo. Como lo menciona Novo (2012), "es posible hablar de verdadero desarrollo en algunas comunidades que, sin crecer económicamente, han sabido reorientar de modo profundo sus prioridades: hacer un consumo equilibrado de sus bienes naturales; distribuir con orientación equitativa los recursos; la educación, etc.” (p. 57)

Ambos autores, Leff y Novo, coinciden en la necesidad de un cambio que va desde lo personal a lo social para alcanzar la meta anhelada; un cambio en la reconstrucción de las valoraciones de cada ser humano, ya que para llegar a la sostenibilidad es ineludible la participación de todos. Como dice Sastre (2011):

Si identificamos, la relación causa-efecto entre un sistema económico y social basado en el consumo y buena parte de los grandes problemas de sostenibilidad a escala mundial, se pueden empezar a realizar acciones que conduzcan al cambio (...) partiendo de lo micro se llega a lo macro (p. 69).

Para Novo (2009a) es necesario afrontar algunas condiciones que permitan la sostenibilidad:

- $\quad$ Reducir el ritmo de consumo de recursos naturales.

- Incentivar el uso de energías renovables.

- $\quad$ Reducir, reciclar y reutilizar los bienes que consumimos.

- $\quad$ Poner énfasis en modelos de agricultura sostenibles. 

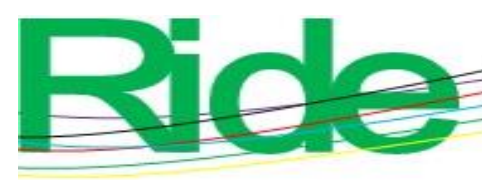

Revista Iberoamericana para la

Investigación y el Desarrollo Educativo

ISSN $2007-7467$

- $\quad$ Hacer una gestión sostenible de los bosques.

- Prestar especial atención a los biomas marinos.

- $\quad$ Invertir grandes recursos en la restauración de ecosistemas degradados.

- Modificar las pautas de alimentación y consumo de los sectores ricos del planeta.

- Cambiar nuestra mentalidad respecto al transporte de mercancías a larga

distancia.

- $\quad$ Evitar el deterioro ambiental que acompaña a la pobreza (p. 215).

Como se puede observar, el desarrollo sostenible requiere de la realización de diferentes actividades que en conjunto favorecen al planeta. Para lograr un cambio es necesario que haya una participación, primeramente, individual; posteriormente vendrán los cambios a nivel local, ya que no hay desarrollo colectivo si no existe desarrollo individual. Es de suma importancia dejar de lado el consumismo y el desarrollismo, pues "la cuestión ambiental ya no es sólo científica, sino política y social. Ya no compete a una minoría: es el patrimonio cotidiano de toda la humanidad" (Novo, 2012, p. 6).

Un proyecto importante que ha trabajado en el campo de sostenibilidad en México es el Grupo Ecológico Sierra Gorda. Sobre este grupo se puede decir lo siguiente:

Trabaja desde 1987 para la protección del medioambiente y el desarrollo sostenible de las comunidades rurales en el estado de Querétaro, en el corazón de México. Su constancia e innovación le ha valido la reciente obtención del prestigioso premio National Geographic World Legacy, en la categoría Conservación del Mundo Natural, que destaca a compañías y organizaciones líderes en viajes y eco-turismo (Organización de las Naciones Unidas para la Educación, la Ciencia y la Cultura [Unesco], 2016).

Desarrollar un proyecto como el antes mencionado no es fácil, requiere de un largo camino que debe ser acompañado por la educación. 


\section{Desarrollo sostenible y educación}

Para Luengo (2004), el término educación alude a lo siguiente:

El término educación tiene un doble origen etimológico (...). Como el verbo latino educere significa conducir fuera de, extraer de dentro hacia fuera, desde esta posición, la educación se entiende como el desarrollo de las potencialidades del sujeto basado en la capacidad que tiene para desarrollarse. (...) El término educare se identifica con los significados de cría, alimentar y se vincula con las influencias educativas o acciones que desde el exterior se llevan a cabo para formar, criar, instruir o guiar al individuo (p 32).

Sin duda habría que tener en cuenta estas dos concepciones del término en el enfoque educativo que pretende lograr la formación para un desarrollo sostenible. La educación implica tanto el recibir de las personas lo que sus capacidades le permitan aportar como el proceso de guía y, las más de las veces, de imposición de contenidos culturales.

En otra posición, Caballero (2011) sostiene lo siguiente:

Enseñar a los participantes a "aprender a aprender" contribuye también al desarrollo integral del ser humano y su desenvolvimiento satisfactorio en el mundo que lo rodea y del que forma parte. Esto quiere decir que el Sujeto pueda comprender, dar significado a las nuevas experiencias y llegar a la resolución de problemas para transformar su entorno (...):

- Aprender a conocer, estimulando conocimientos e información relevante y pertinente para la re-significación de su estructura cognitiva.

- $\quad$ Aprender a hacer, propiciando el desarrollo de competencias y habilidades para su aplicación en la cotidianeidad.

- Aprender a ser, reconociendo valores, ambientes, y encaminando al sujeto a que los asimile e incorpore en su actuar cotidiano para la modificación de sus conductas.

- $\quad$ Aprender a convivir, favoreciendo la formación de grupos en el que el ser humano se reconozca como actor social que socializa e interactúa con los demás para transformar su entorno (p. 50). 

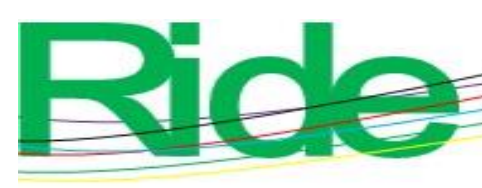

Revista Iberoamericana para la

Investigación y el Desarrollo Educativo

ISSN 2007 - 7467

Leff (2011), por su parte y desde una perspectiva de la pedagogía para el desarrollo sustentable mediante la educación, menciona lo puesto a continuación:

No sólo se trata de informar sobre la crisis ambiental y el calentamiento global, sino de desentrañar sus causas profundas. Es una práctica pedagógica, que más que impartir al alumno los conocimientos actuales y las normas sociales (y ambientales) (...), es preparar el pensamiento y la vida para lo incógnito, para pensar lo impensado, para desconstruir teórica y prácticamente el mundo, para ensayar otros modos de pensar-sentir-actuar, para escuchar lo inefable, para mirar lo otro intraducible a mi yo y al uno mismo; para enlazarse en un diálogo de saberes, donde la fecundidad no brota del juicio de la verdad probada, sino del por-venir probable (p. 102).

En esa misma línea, para Novo (2009b) “es necesario que la educación, como instrumento de socialización y de actitud crítica, adopte respuestas válidas para los retos que tiene planteados la humanidad" (p. 197). En suma, llevar a cabo las pautas que rigen al desarrollo sostenible es más fácil si se inicia desde las aulas con los estudiantes a nivel general.

En este sentido, y en particular en lo que toca a la educación superior, existe la necesidad de que las universidades se involucren de manera comprometida con los problemas que afectan la viabilidad del planeta como un sistema de soporte de vida de los seres humanos y de todas las especies vivas (Conde, González y Mendieta, 2006, p. 15).

La educación universitaria tendrá que tomar en cuenta que "el pensamiento global debe prolongarse en una acción local, acción que comprometa al individuo con su entorno. De modo que conviene que se trabaje ayudando a comprender los problemas desde su realidad cotidiana, allí donde lo real les interpela" (Novo, 2012, p. 74). Así, pues, no se puede intervenir en situaciones que están lejos de nuestro alcance, más bien se requiere tomar medidas en situaciones que afectan directamente y con las personas más próximas, en cambiar hábitos y modos de pensar.

Lo anterior alude a vivir una ética como la construcción de un ethos. Para Mendoza (2016), “el sentido originario de la ética consiste en 'encaminar', acompañar la formación de cada ser humano, desde sí mismo y para el mundo, con el objetivo de asumir el sentido de la 

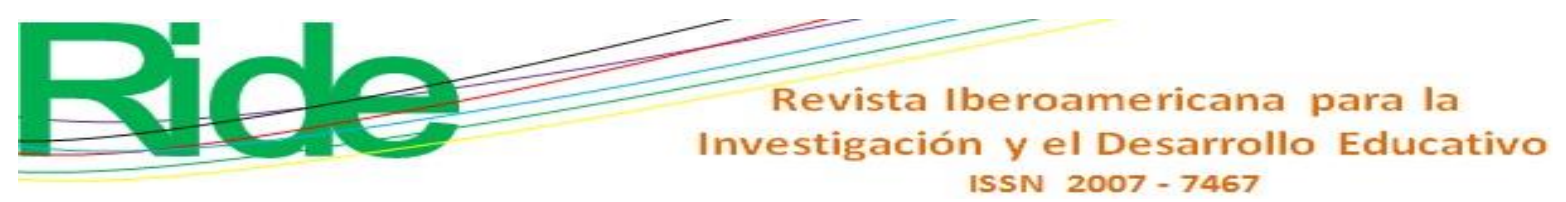

convivencia (con-vivencia) con el otro, co-munidad (común unidad)" (p. 12). Desde el inicio ya se forma parte de un todo, no se vive de manera aislada; se vive en un conjunto en el cual las acciones de unos repercuten en los otros y viceversa. Es por esto por lo que se menciona que la construcción del ethos va desde lo individual a lo social, se construye con base en las acciones que se deciden hacer o no hacer, todo está conectado de algún manera con los otros. De nueva cuenta en palabras de Mendoza (2016):

Cada grupo o comunidad y/o cultura grupal tienen un modo de pensar el ethos Originario y de orientarlo. Eso depende de su visión del mundo. A esa visión interpretativa del ethos se le denomina ética, como una reflexión sobre la posibilidad originaria del ser humano. Tal visión interpretativa conduce posteriormente a la concepción de ciertos códigos de normas y leyes, para guiar el ethos comunitario, es decir, el modo de ser de las acciones de la comunidad (p. $14)$.

Es así como el ethos se va formando de acuerdo a las costumbres y tradiciones de la sociedad en que se encuentre inmerso cada individuo y es trabajo de cada uno construir su propio destino y ayudar a que las generaciones venideras aprendan y realicen acciones responsables para el cuidado del medio. García Rendón y Panchi (2015) lo plantean en los siguientes términos:

Es necesario vivir la construcción del ethos más allá de la morada humana. Pasar de una visión antropocentrista a una que involucre a todos los actores que intervienen en la vida. Así como asumir la co-responsabilidad de las consecuencias que se tienen por las acciones que ejercemos en detrimento de los Otros seres vivos (p. 176)

Desde este punto de vista, se entiende que "el ethos es el proceso de formación y de construcción de la identidad sociomoral" (Yurén Camarena, 2001, p. 99). Viene desde la propia condición humana, es desde el interior de cada persona que nace el bien o el mal, y es decisión de la misma darle el valor que considere al entorno en el que se encuentra.

Sobre esta base se puede decir que los seres humanos se van construyendo día con día, van adquiriendo nuevos conocimientos y es decisión de cada ser humano construirse a favor de sí mismos y de los otros. Porque, como es bien sabido, no todas las acciones o conocimientos 

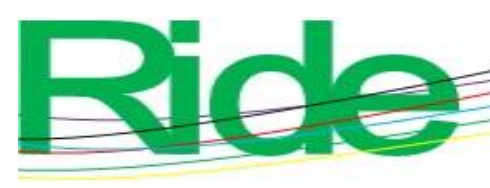

Revista Iberoamericana para la

Investigación y el Desarrollo Educativo

ISSN $2007-7467$

ayudan a formar personas responsables con el ambiente; todo va a depender de las valoraciones que se les asigne al entorno.

Para Benhabib (García Rendón, 2016), "lo valorativo concierne a lo que individual y colectivamente se considera valioso, merecedor de nuestro esfuerzo y esencial a la felicidad humana” (p. 272). Asimismo, Martínez (García Rendón, 2016) sostiene lo siguiente:

El valor es la significación socialmente positiva de los objetos y fenómenos de la realidad, mientras que la valoración no es más que el reflejo subjetivo en la conciencia de la persona de la significación que para ella poseen los objetos y fenómenos de la realidad (p. 272).

Cada ser humano asigna diferentes valoraciones al entorno en el cual se encuentra, ya que cada persona crece y es guiado de diferentes maneras, todo de acuerdo a la sociedad en la cual se encuentra inmerso. Es en este momento en el que es ineludible la participación de las familias (como primer círculo social) para que dichas valoraciones estén a favor de las relaciones afectivas sanas y el cuidado del entorno.

El ser humano, teniendo en cuenta los valores que lo guían y las valoraciones que posee, comienza a construir su ethos, lo cual posteriormente lo caracterizará como un ser humano ético. Al respecto, García Rendón y Panchi (2015) mencionan:

El ser humano es ético cuando renuncia a estar sobre los otros para estar y ser solidario con los otros, cuando es capaz de entender las exigencias del equilibrio ecológico y poner a sí mismo límites a los deseos antropocéntricos que tiene (p. 160).

La satisfacción de nuestras necesidades y la satisfacción de nuestros deseos van ligados a las valoraciones que posee cada ser humano: si nuestras valoraciones están basadas en principios de vida sostenibles existirán grandes posibilidades de dejar un mundo sano para las futuras generaciones y garantizar una buena calidad de vida. 


\section{Hacia la construcción de un ethos sostenible}

De acuerdo con Leff (2011), "pensar el futuro es desconstruir a la economía, que desconoce a la naturaleza y que descuenta el futuro" (p. 95). Y para lograr esto es necesaria la sensibilización de cada una de las sociedades, para que conozcan que el crecimiento desmedido no lleva a ningún lado, que está en cada ser humano tomar las medidas necesarias para proteger la naturaleza y dejar un futuro productivo a las nuevas generaciones. Puesto que los seres humanos "desde que nos levantamos hasta que nos acostamos, nuestra vida se basa en la elección entre pequeñas acciones cotidianas que pueden marcar la diferencia entre un comportamiento sostenible y uno totalmente insolidario" (Sastre, 2011, p. 68).

Asimismo, Novo propone desarrollar una conciencia acerca de las formas de vida con las cuales se comparte el planeta. Este modelo de desarrollo debe abarcar todos los ángulos posibles: "los aspectos éticos, las formas de comunicación, las aptitudes y actitudes vinculadas a los afectos, los sentimientos, que dan sentido a las conductas individuales y colectivas" (Novo, 1996, p. 96).

Al respecto, Leff (2011) comenta lo siguiente:

Se trata de construir los significados que abran las vías para la fundación de nuevos modos de pensar, de sentir y de ser; que más allá de servir al establecimiento de nuevas normas ambientales - de una ética y una moral que establezca lo permitido y lo prohibido ante la naturaleza-, forjen los referentes y objetivos de nuevos derechos humanos hacia el ambiente y hacia una vida sustentable y con sentido. Se trata de reavivar la palabra para reinventar identidades y revivir los movimientos sociales por la reapropiación de la naturaleza y de sus culturas (p. 96).

Es necesaria una educación que permita el crecimiento personal y considere crear cambios en los modos de hacer las cosas.

Se hace necesario un consenso global encaminado a la búsqueda de ese nuevo modelo de desarrollo que suscite profundos cambios en los actuales estilos de vida, de formas de pensar, de valores éticos y morales... y todo ello sólo es posible si surge desde procesos educativos que promuevan la sensibilización, la 


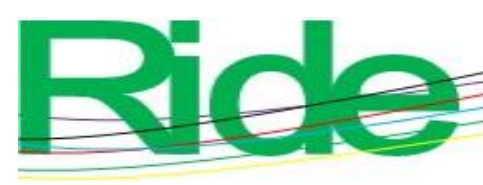

Revista Iberoamericana para la Investigación y el Desarrollo Educativo ISSN $2007-7467$

concienciación, la participación y el desarrollo de actitudes y capacidades más responsables en todos los ámbitos de la sociedad (Dueñas, 2011, p. 16).

Consecuentemente, las acciones de cada ser humano serán guiadas por el respeto y la tolerancia, porque de nada sirve preparar a las generaciones venideras e inculcarles grandes conocimientos si en un futuro no tendrán un planeta sano en el cual vivir.

Una característica del desarrollo sostenible "es el hecho de que se inicia desde las bases educativas" (Novo, 1996, p. 77), ya que no se puede esperar que la sociedad cuide del medio si no ha sido educada para tal fin, si los individuos no poseen los conocimientos necesarios para cuidarlo; a la vez, es importante inculcar valores y trabajar en las valoraciones que den prioridad al medio que funge como sustento.

En México, de acuerdo a Conde, González y Mendieta (2006):

Desde un principio, los programas nacionales de Educación y del Medio Ambiente establecieron la urgente necesidad de incorporar la perspectiva ambiental en la educación superior a fin de generar los cambios internos requeridos para fomentar que las instituciones de educación superior (IES) ofrezcan respuestas pertinentes a los problemas ambientales, para que sean partícipes activas en la construcción de escenarios deseables de desarrollo (p. 18).

Y siguiendo a Dueñas (2011), la educación para el desarrollo sostenible "debe ser considerada como una herramienta pedagógica de transmisión de nuevos modelos éticos y actitudinales hacia los ciudadanos para que estos se sientan partícipes del cambio, para que ellos mismos se constituyan en el cambio" (p. 15).

Por ello se hace hincapié en la construcción de valores orientados hacia el desarrollo sostenible, para crear movimientos con el fin de que esta sociedad se convierta en una sociedad responsable.

Es prioritario desarrollar valores para la convivencia que permitan desarrollar las capacidades de plantear, identificar y diseñar la posible solución de problemas; que aspiren a alcanzar un desarrollo donde generar conocimientos se traduzca en formas de producción y consumo racionales y sustentables: en trabajo creativo, en amplios canales de acceso a los bienes públicos, en mejores condiciones de 

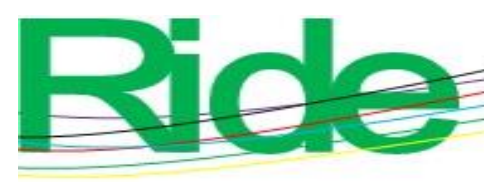

Revista Iberoamericana para la

Investigación y el Desarrollo Educativo

ISSN 2007 - 7467

expresión social, el respeto a la diversidad cultural y el cuidado a las personas (Peza, 2011, p. 64).

Los seres humanos le han asignado y asignan valores a su entorno y a las personas que lo rodean, ya que, como se mencionó anteriormente, el ser humano es parte de un todo, forma parte de un espacio todo afecta a todos.

El hecho de tener en cuenta los esquemas previsores o marcos de referencia (Eisner 1897) de los sujetos que aprenden contribuye notablemente no sólo a la adquisición de nuevos conocimientos o habilidades, sino también, en muchos casos, a lograr una verdadera reorganización de su trama cognitiva y afectivo valorativa del entorno que les es propio y de su papel activo en el mismo (Novo, 2005, p. 157).

Cuando una persona actúa y vive de forma responsable hace más fácil la tarea de trabajar en conjunto con otros, al mismo tiempo que inculca y esparce los valores por los cuales dicha persona se rige.

Se trata de que cada grupo que enseña y cada grupo que aprende tengan la oportunidad de revisar sus valores, someterlos a crítica, y elucidar valores nuevos que permitan avanzar en la dirección de la equidad social y el equilibrio ecológico (Novo, 1996, p. 96).

De esta manera, se crean nuevos conocimientos que propician el cuidado del medio ambiente, se forma una cadena de ayuda, permitiendo a cada persona "verificar la pertinencia de sus conocimientos y habilidades en contextos reales y adherirse a valores que se expresen mediante un compromiso efectivo con el entorno" (Novo, 2005, p. 153).

Aunado a lo anterior, casi al centro de la ecuación, se encuentra la importancia de trabajar primero a nivel personal para que, posteriormente, se pueda colaborar a nivel grupal. Así, se crean grupos en los cuales "es posible trabajar en procesos que implican la construcción colectiva de conocimiento, el debate conjunto de valores, la asunción grupal de responsabilidad" (Novo, 2005, p. 153). Todo lo cual se convierte en una caja de herramientas para un mundo mejor, un mundo que se encuentra en constante cambio.

En resumen, se insiste en la importancia de la sostenibilidad y en la secuencia que dicta que desde lo micro se hará que en lo macro se pueda alcanzar el cambio, esto es, tener un modo 

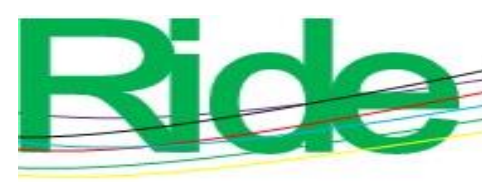

Revista Iberoamericana para la Investigación y el Desarrollo Educativo ISSN $2007-7467$

de vida sostenible provoca que otras personas tengan la posibilidad de satisfacer sus necesidades. Por todo ello, es importante conocer a los estudiantes universitarios; y empezar a hacerlo por los valores aprendidos y las valoraciones que se rigen sus acciones en relación con el desarrollo sostenible.

\section{Método}

La presente investigación fue de corte cualitativo. Se fundamenta en una perspectiva interpretativa. Para simplificar la organización, el análisis y la interpretación de datos, el procesamiento de la información se realizó a través del software científico ATLAS.ti. 8.

Se consideraron dos categorías: 1) construcción del ethos y 2) educación sostenible. Dentro de esta fase de análisis, se realizaron anotaciones para las citas y códigos con la finalidad de vincular la teoría con las respuestas de los participantes.

La información obtenida de las entrevistas se codificó y se clasificó de acuerdo a las categorías expuestas en el marco teórico y a las dos categorías arriba mencionadas. Posteriormente, se comparó la información en el software de análisis cualitativo y, finalmente, se analizó la información de las entrevistas en conjunto con la información teórica: se realizaron constantes comparaciones entre la información teórica y la obtenida de las entrevistas para detectar puntos de vista en común entre los participantes y la teoría.

\section{Muestra}

Se realizaron en total 47 entrevistas semiestructuradas a estudiantes de las facultades de Medicina, Enfermería, Ingeniería, Arquitectura y Ciencias de la Conducta.

\section{Resultados}

A continuación se muestran las preguntas y las categorías que emergieron con base en las respuestas de los entrevistados y posteriormente el número de entrevista correspondiente. 


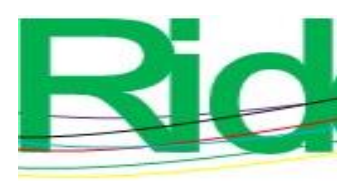

Revista Iberoamericana para la Investigación y el Desarrollo Educativo ISSN $2007-7467$

Tabla 1. Resultados de la categoría de construcción del ethos sostenible

\begin{tabular}{|c|c|c|}
\hline Pregunta & $\begin{array}{l}\text { Categorías emergentes de acuerdo a } \\
\text { las respuestas de los entrevistados }\end{array}$ & Núm. de entrevista \\
\hline \multirow[t]{5}{*}{$\begin{array}{l}\text { ¿Qué acciones } \\
\text { realizaba su familia } \\
\text { respecto al cuidado } \\
\text { del ambiente? }\end{array}$} & $\begin{array}{l}\checkmark \text { Separar/Reciclar/Recolectar la } \\
\text { basura }\end{array}$ & $\begin{array}{l}\text { E. } 3,4,5,6,7,8,11,12,14, \\
39,17,20,27,28,29,40,22, \\
23,24,25,26,30,32,34,35, \\
36,38,41,42,43\end{array}$ \\
\hline & $\begin{array}{l}\checkmark \text { Ahorrar/Reciclar/Cuidar el } \\
\text { agua }\end{array}$ & $\begin{array}{l}\text { E. } 2,5,7,9,14,32,35,38,41 \text {, } \\
42,47\end{array}$ \\
\hline & $\checkmark \quad$ Ahorrar luz & E. $5,30,31,43$ \\
\hline & $\checkmark \quad$ Hacer compostas & E. 10,13 \\
\hline & $\begin{array}{ll}\checkmark & \text { Otras/ ninguna }\end{array}$ & $\begin{array}{l}\text { E. } 1,16,19,44,45,46,18,21 \text {, } \\
33,37\end{array}$ \\
\hline \multirow[t]{5}{*}{$\begin{array}{c}\text { De esas acciones, } \\
\text { ¿cuáles realiza usted? }\end{array}$} & $\begin{array}{l}\checkmark \text { Separar/Reciclar/Recolectar } \\
\text { basura }\end{array}$ & $\begin{array}{l}\text { E. 1, 4, 8, 9, 23, 27, 29, 36, 40, } \\
3,5,6,10,11,12,13,14,15, \\
39,16,18,19,20,21,22,24, \\
25,26,28,30,31,32,33,34, \\
37,38,42,44,45,46\end{array}$ \\
\hline & $\checkmark \quad$ Recolección de PET & E. $13,14,15,33,35,41$ \\
\hline & $\begin{array}{l}\checkmark \text { Ahorrar/Reciclar/Cuidar el } \\
\text { agua }\end{array}$ & $\begin{array}{l}\text { E. } 2,7,14,17,30,35,37,38 \text {, } \\
42,43,47\end{array}$ \\
\hline & $\checkmark \quad$ Ahorrar luz & E. $17,30,31,43$ \\
\hline & $\begin{array}{ll}\checkmark & \text { Hacer compostas }\end{array}$ & E. 10,11 \\
\hline \multirow[t]{2}{*}{$\begin{array}{c}\text { ¿Qué acciones realiza } \\
\text { respecto al cuidado } \\
\text { de otras personas? }\end{array}$} & $\begin{array}{ll} & \text { Actúo de forma } \\
& \text { solidaria/Respetuosa }\end{array}$ & $\begin{array}{l}\text { E. } 1,8,10,12,39,18,21,22, \\
23,24,25,27,32,33,34,35 \text {, } \\
37,38,42,43\end{array}$ \\
\hline & $\begin{array}{l}\checkmark \text { Mantener la limpieza de } \\
\text { entorno }\end{array}$ & E. $4,11,13,15,16,29,47$ \\
\hline
\end{tabular}




\begin{tabular}{|c|c|c|}
\hline & $\begin{array}{ll}\checkmark & \text { Hablar con la gente sobre no } \\
\text { tirar basura/Fomentar la cultura } \\
\text { del cuidado del ambiente }\end{array}$ & E. $2,3,14,15$ \\
\hline & $\checkmark \quad$ Ninguna & $\begin{array}{l}\text { Е. } 5,6,7,9,19,20,26,28,30, \\
31,36,40,41,44,45,46\end{array}$ \\
\hline \multirow[t]{2}{*}{$\begin{array}{l}\text { ¿A quiénes considera } \\
\text { como responsables } \\
\text { para el cuidado del } \\
\text { ambiente? }\end{array}$} & $\checkmark$ A todos & $\begin{array}{l}\text { E. 1, 2, 3, 4, 5, 6, 7, 8, 9, 10, } \\
\text { 11, 12, 13, 14, 15, 16, 18, 19, } \\
20,21,22,23,24,25,26,27, \\
28,29,30,31,32,33,34,35, \\
36,37,38,39,40,41,42,43, \\
44,45,46,47\end{array}$ \\
\hline & $\checkmark \quad$ Al gobierno & E. 17 \\
\hline
\end{tabular}

Fuente: Elaboración propia

Tabla 2. Resultados de la categoría de educación sostenible

\begin{tabular}{|c|c|c|}
\hline Pregunta & $\begin{array}{c}\text { Categorías emergentes de acuerdo a las } \\
\text { respuestas de los entrevistados }\end{array}$ & Núm. de entrevista \\
\hline \multirow[t]{6}{*}{$\begin{array}{l}\text { ¿Qué es para } \\
\text { usted la } \\
\text { sostenibilidad? }\end{array}$} & $\checkmark \quad$ No sabe & $\begin{array}{l}\text { E. } 2,3,4,6,7,12,18,20, \\
21,22,27,30,32,35,36 \text {, } \\
38,42,44,45,46,24\end{array}$ \\
\hline & $\checkmark$ Cuidar & E. $8,10,13$ \\
\hline & $\checkmark$ Administrar recursos & $\begin{array}{l}\text { E. 9, 17, 23, 28, 31, 33, 34, } \\
37\end{array}$ \\
\hline & $\checkmark$ Reciclar & E. 11,14 \\
\hline & $\checkmark \quad$ Equilibrio/estabilidad & E. 16,41 \\
\hline & $\checkmark$ Otras & $\begin{array}{l}\text { E. } 1,5,15,39,19,25,26 \text {, } \\
29,40,43,47\end{array}$ \\
\hline $\begin{array}{l}\text { ¿Cómo piensa } \\
\text { usted que sería }\end{array}$ & $\checkmark$ No sabe & $\begin{array}{l}\text { E. } 2,5,7,44,45,46,4,12 \text {, } \\
18\end{array}$ \\
\hline
\end{tabular}




\begin{tabular}{|c|c|c|c|}
\hline \multirow{6}{*}{$\begin{array}{l}\text { un modo de } \\
\text { vida } \\
\text { sostenible? }\end{array}$} & \multicolumn{2}{|c|}{$\checkmark$ Usar energías renovables } & $\begin{array}{l}\text { E. } 3,9,10,15,22,28,30 \text {, } \\
31,23\end{array}$ \\
\hline & \multicolumn{2}{|c|}{$\begin{array}{ll}\checkmark & \text { Reciclar/Reutilizar }\end{array}$} & E. $11,13,14,19,34$ \\
\hline & \multicolumn{2}{|c|}{$\checkmark \quad$ Disminuir el consumismo } & E. $16,21,26,37,40$ \\
\hline & \multicolumn{2}{|c|}{$\checkmark \quad$ Administrando recursos } & E. $28,31,33,37$ \\
\hline & \multicolumn{2}{|c|}{$\checkmark \quad$ Cuidar } & E. $36,41,42$ \\
\hline & \multicolumn{2}{|c|}{$\checkmark$ Otras } & $\begin{array}{l}\text { E. } 1,6,39,17,20,24,25, \\
27,29,32,35,38,43,47\end{array}$ \\
\hline \multirow{6}{*}{$\begin{array}{c}\text { ¿Qué tan } \\
\text { importante es } \\
\text { para usted } \\
\text { cuidar del } \\
\text { ambiente para } \\
\text { el futuro de las } \\
\text { próximas } \\
\text { generaciones? }\end{array}$} & \multirow{5}{*}{ 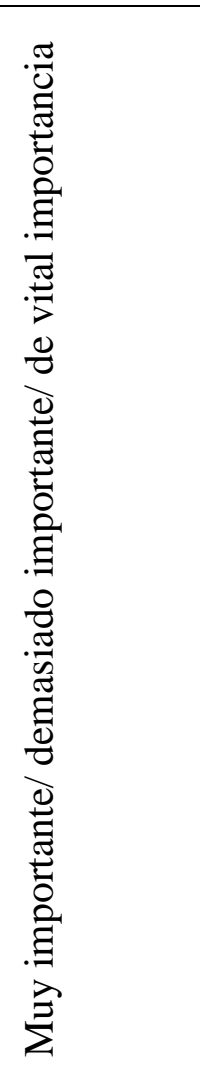 } & $\begin{array}{l}\text { Aumenta la } \\
\text { contaminación }\end{array}$ & E. 1,41 \\
\hline & & $\begin{array}{ll}\checkmark & \text { Preservar la naturaleza } \\
& \text { para las próximas } \\
& \text { generaciones }\end{array}$ & $\begin{array}{l}\text { E. } 2,3,6,8,9,10,13,16, \\
17,19,21,23,26,29,31, \\
33,35,36,37,38,47,43, \\
45,32,39,28,15,22,20, \\
42,12,11\end{array}$ \\
\hline & & $\begin{array}{l}\checkmark \text { Para tener una mejor } \\
\text { calidad de vida }\end{array}$ & E. $14,34,47$ \\
\hline & & $\begin{array}{l}\checkmark \text { Aumentan los problemas } \\
\text { de salud }\end{array}$ & E. $30,39,46$ \\
\hline & & $\checkmark \quad$ Otras respuestas & E. $7,18,25,27,40,4,5,24$ \\
\hline & $\begin{array}{l}\text { Poco } \\
\text { importante }\end{array}$ & $\checkmark \quad$ Poco importante & E. 44 \\
\hline \multirow{2}{*}{$\begin{array}{c}\text { ¿Qué acciones } \\
\text { considera que } \\
\text { debería de } \\
\text { realizar la }\end{array}$} & \multicolumn{2}{|c|}{$\begin{array}{ll}\checkmark & \text { Brigadas/Campañas }\end{array}$} & $\begin{array}{l}\text { E. } 1,3,4,5,6,7,8,10,11 \text {, } \\
17,20,31,40,45\end{array}$ \\
\hline & \multicolumn{2}{|c|}{$\checkmark$ Ahorrar energía eléctrica } & E. $9,8,25,47$ \\
\hline
\end{tabular}




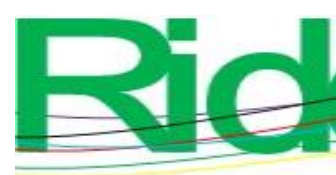

Revista Iberoamericana para la Investigación y el Desarrollo Educativo ISSN 2007 - 7467

\begin{tabular}{|c|c|c|}
\hline \multirow{6}{*}{$\begin{array}{c}\text { universidad } \\
\text { respecto al } \\
\text { cuidado del } \\
\text { ambiente? }\end{array}$} & $\begin{array}{l}\checkmark \text { Aumentar la cantidad de botes de } \\
\text { basura/Ceniceros }\end{array}$ & $\begin{array}{l}\text { E. } 12,13,14,18,26,30,33 \text {, } \\
34,35,37,42,46\end{array}$ \\
\hline & $\checkmark$ Ahorrar agua & E. $15,24,47$ \\
\hline & $\checkmark$ Difusión de la información & $\begin{array}{l}\text { E. } 2,19,23,24,28,29,32, \\
43\end{array}$ \\
\hline & $\begin{array}{l}\checkmark \text { Inclusión de materias en la curricula para } \\
\text { el cuidado del ambiente }\end{array}$ & E. 39,33 \\
\hline & $\checkmark$ Penalizar/Incentivar & E. 16,37 \\
\hline & $\checkmark$ Darle mantenimiento a la universidad & E. $21,22,27,36,38,41,44$ \\
\hline \multirow[t]{2}{*}{$\begin{array}{c}\text { ¿Qué hace } \\
\text { usted con la } \\
\text { basura (cartón, } \\
\text { papel, } \\
\text { desperdicios } \\
\text { de comida, } \\
\text { envases, etc.)? }\end{array}$} & $\begin{array}{ll}\checkmark \text { Reciclar/Reutilizar/Separar/Clasificar } \\
\end{array}$ & $\begin{array}{l}\text { E. 2, 3, 4, 5, 6, 7, 8, 9, 10, } \\
12,13,14,15,16,39,18, \\
18,21,22,23,24,25,27, \\
28,29,30,31,32,33,34, \\
35,36,37,38,40,41,42, \\
43,44,45,46,47\end{array}$ \\
\hline & $\checkmark \quad$ Desperdicios para abono/Compostas & $\begin{array}{l}\text { E. } 1,2,3,16,17,20,21,26 \text {, } \\
29\end{array}$ \\
\hline \multirow[t]{5}{*}{$\begin{array}{l}\text { ¿Realiza algún } \\
\text { tipo de } \\
\text { actividad para } \\
\text { el cuidado del } \\
\text { agua y la } \\
\text { energía? }\end{array}$} & $\checkmark$ Ahorrar/Reciclar el agua & $\begin{array}{l}\text { E. 1, 3, 5, 7, 8, 9, 10, 11, 12, } \\
13,14,15,39,16,17,18, \\
19,20,21,23,24,25,26, \\
28,29,30,31,32,34,42, \\
45,47\end{array}$ \\
\hline & $\checkmark \quad$ Apagar la luz que no se usa & $\begin{array}{l}\text { E. } 2,3,11,39,17,18,19, \\
20,21,23,24,26,28,32 \text {, } \\
37,38,42,43,45,46,47\end{array}$ \\
\hline & $\checkmark \quad$ Utilización de focos ahorradores & E. $30,31,33$ \\
\hline & $\begin{array}{ll}\checkmark & \text { Desconectar aparatos eléctricos }\end{array}$ & E. $39,26,28,29,38,41,46$ \\
\hline & $\checkmark \quad$ Ninguna & E. $4.6,22,35,36,40,44$ \\
\hline ¿Qué aspectos & $\checkmark$ La economía & E. $5,7,6,14,15$ \\
\hline
\end{tabular}




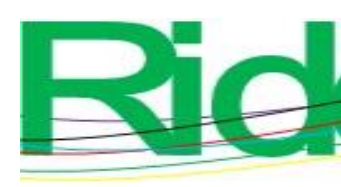

Revista Iberoamericana para la Investigación y el Desarrollo Educativo ISSN $2007-7467$

\begin{tabular}{|c|c|c|}
\hline \multirow{5}{*}{$\begin{array}{l}\text { toma en cuenta } \\
\text { cuando se trata } \\
\text { de su } \\
\text { alimentación? }\end{array}$} & $\checkmark$ Evitar alimentos con envolturas & E. 12 \\
\hline & $\checkmark$ Comer a la hora indicada & E. 39,33 \\
\hline & $\checkmark \quad$ Los nutrientes & $\begin{array}{l}\text { E. } 15,18,27,35,36,40,42 \text {, } \\
47\end{array}$ \\
\hline & $\checkmark \quad$ Las proporciones y el balance & E. $30,31,32,33,43,46$ \\
\hline & $\begin{array}{ll}\checkmark & \text { Otras/Ninguna }\end{array}$ & $\begin{array}{l}\text { E. } 1,2,3,4,9,10,22,28, \\
34,41,8,11,13,16,17,19, \\
20,21,23,24,25,26,29, \\
37,38,44,45\end{array}$ \\
\hline
\end{tabular}

Fuente: Elaboración propia

\section{Discusión}

El objetivo de este proyecto fue identificar y analizar las percepciones y acciones relacionadas con el modo de vida sostenible o no sostenible en estudiantes de la UAEMéx, Campus Toluca, tomando en consideración dos categorías: 1) construcción del ethos sostenible y 2) la educación sostenible.

Retomando la definición de González (1996), el ethos refiere a lo siguiente:

Ethos como "lugar" humano de "seguridad" existencial. Aunque también lo significativo es que se trate de un lugar acostumbrado, habitual, familiar. De ahí que ethos signifique también costumbre. Remite a una forma habitual de comportamiento. Es un modo habitual, continuo, de comportarse, de ser en el tiempo; forma de estabilidad y persistencia temporal (p. 10).

Los datos obtenidos (Tabla 1) concuerdan con esta definición en el sentido de que las acciones que mencionan los entrevistados son las que tradicionalmente realizaban sus familias con anterioridad. De las entrevistas, resaltan las acciones de separar, reciclar y recolectar basura como práctica tradicional y llevadas a cabo por las familias de los entrevistados. Y estas acciones son las mismas que realizan actualmente, aunadas a la recolección de tereftalato de polietileno (PET, por sus siglas en inglés), el ahorro y la reutilización del agua y el ahorro de energía eléctrica. 


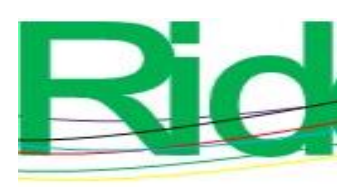

Revista Iberoamericana para la

Investigación y el Desarrollo Educativo

ISSN 2007 - 7467

Para llegar a la meta de la sostenibilidad es ineludible tener en cuenta que cada ser humano forma parte de un todo, que mantiene relaciones de interdependencia. Por ende, se les preguntó a los entrevistados respecto a que si realizan algún tipo de acción en cuanto al cuidado de otras personas. Nadie mencionó alguna acción como tal; mencionaron que más bien tienen un comportamiento respetuoso y solidario con los demás. Y en ese mismo rubro, algo que llama particularmente la atención es que fueron pocos los entrevistados que respondieron que al mantener la limpieza de su entorno o al hablar con los conocidos para fomentar el cuidado del ambiente están contribuyendo al cuidado de los otros.

Aunado a lo anterior, podemos probar con la información obtenida que los entrevistados tienen conciencia de que el cuidado del ambiente no es responsabilidad de una sola persona o ente u organización; más bien saben que cada uno de los que viven en el planeta son responsables de alguna manera por el desgaste del mismo, que todos tienen esa responsabilidad de cuidarlo, que al final todos son un equipo para preservar lo que los mantiene con vida.

Por otro lado, la categoría de sostenibilidad para el Programa de las Naciones Unidas para el Medio Ambiente (PNUMA) (Organización de las Naciones Unidad [ONU], 2015) apunta a lo siguiente:

El desarrollo sostenible, desde un enfoque antropocéntrico, se refiere, a la necesidad de utilizar los recursos en el presente de modo que el planeta pueda satisfacer, las necesidades de las generaciones futuras. Desde un punto de vista biocéntrico significa, que se respete el equilibrio de los sistemas que albergan la vida; la capacidad de carga de los ecosistemas; los umbrales de absorción de los sumideros, etc. (p. 8).

En ese sentido, es bastante llamativo que las respuestas obtenidas (Tabla 2) por los entrevistados al preguntarles por la definición de sostenibilidad no concuerden con las respuestas de cuando se les preguntó que cómo piensan que sería un modo de vida sostenible. Por lo que se puede concluir que los estudiantes no tienen conocimiento del concepto como tal, pero sí saben qué tipo de acciones pueden realizar para llegar a la sostenibilidad.

En la definición del concepto sostenibilidad se habla acerca de la satisfacción de necesidades del ser humano, pero también de la satisfacción de necesidades de las generaciones futuras, es por ello por lo que una de las preguntas va encaminada hacia esta dirección. Y con 


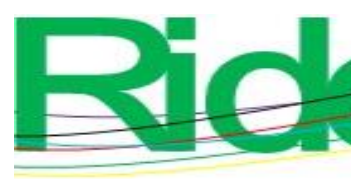

Revista Iberoamericana para la

Investigación y el Desarrollo Educativo

ISSN 2007 - 7467

base en las respuestas de los entrevistados, es lícito afirmar que los universitarios son conscientes de que el planeta puede satisfacer sus necesidades, así como de que es necesario preservarlo para la satisfacción de las necesidades de las futuras generaciones. En suma, los entrevistados concuerdan en que es muy importante cuidar del ambiente, ya que consideran que al cuidar de él se mejorará su calidad de vida, disminuirá la contaminación y los problemas ambientales.

Respecto a las preguntas acerca de las acciones que realizan entrevistados en cuanto a la basura, se puede constatar que gran parte de ellos emplean acciones a favor del cuidado del ambiente: reciclan, separan, reutilizan y clasifican la basura cotidianamente. También mencionaron, que estas acciones son difíciles de realizar cuando se encuentran dentro del campus universitario, ya que dentro del mismo no existen botes de basura o son insuficientes para llevar a cabo dichas acciones.

En cuanto a las actividades para el cuidado del agua y la energía, las acciones más comunes son las del ahorro y la reutilización del agua, así como las de apagar las luces que no se ocupan y desconectar los aparatos eléctricos que no están en funcionamiento. Dichas acciones son las mismas que se tratan de llevar a cabo dentro del campus universitario, apagando las luces de los salones que no están en uso, o reportando si existe algún tipo de desperdicio de agua.

Por último, en lo referente a los aspectos que toman en cuenta los universitarios cuando se trata de su alimentación, sobresale que casi la mitad de los entrevistados no toman en cuenta ninguno, ya que la situación económica en la que se encuentran difícilmente les permite adquirir alimentos balanceados y nutritivos; comúnmente suelen comer lo que cuesta menos o lo que los mantenga satisfechos por más tiempo, dejando de lado la importancia de las horas destinadas para cada alimento del día o si los alimentos que consumen aportan nutrientes o no.

\section{Conclusiones}

Se puede identificar que el ser humano ha contribuido a la crisis ambiental que se vive actualmente a través del uso indiscriminado de los recursos naturales y la falta de cuidado al medio ambiente. Los modelos de desarrollo basado en la explotación de los recursos para el bienestar humano han acarreado un sinfín de consecuencias que ponen en riesgo a la vida misma en el planeta. 


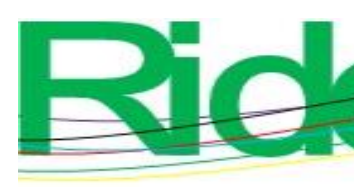

Revista Iberoamericana para la

Investigación y el Desarrollo Educativo

ISSN 2007 - 7467

Es necesario hacer una revisión a las necesidades del ser humano y su prioridad para satisfacerlas, ya que estas, en muchos casos, propician el abuso hacia los recursos y hacia otros seres vivos. Hay que tomar en cuenta que cada acción realizada genera una consecuencia; considerar que, como seres humanos inmersos en situaciones de interdependencia, lo que suceda en determinado lugar del planeta repercute en otro, y que lo mejor para todos es que esas consecuencias afecten lo menos posible al medio, que ha brindado todo para poder vivir. En pocas palabras, propiciar el desarrollo sostenible.

Lo anterior enfatiza la importancia de la participación de cada ser humano; en las acciones que pueden marcar el cambio de una sociedad, empezando por el análisis de los valores y las valoraciones que guían nuestras acciones. Este desarrollo sostenible permitirá solventar las necesidades actuales sin comprometer las de las futuras generaciones, así como preservar los recursos naturales. Se puede decir que es necesario contar con dos valores importantes, al menos: 1) la responsabilidad, que nos permite la sensibilización a las problemáticas actuales y responder por nuestras acciones y 2) la solidaridad, que refleja el compromiso con el presente y el futuro de las comunidades en el desarrollo sostenible.

Las universidades, por su parte, tienen un papel muy importante en la sociedad. Ahí se forman a los individuos que, tanto a lo largo de su formación como al terminar sus estudios, tendrán la posibilidad de influir en su entorno familiar, universitario y social.

Por todo lo anterior, se consideró importante realizar la investigación en la UAEMéx, Campus Toluca; conocer qué percepciones y acciones tienen los alumnos con relación al desarrollo sostenible. Con base en las respuestas, se pueden resaltar cuatro aspectos. El primero, los estudiantes reconocen que la crisis ambiental es causa de los seres humanos, por lo tanto, la recuperación del mismo y sus cuidados son responsabilidad de los mismos quienes la han causado. Segundo, efectivamente, los universitarios realizan acciones sostenibles, que provienen de hábitos y costumbres adquiridos en su entorno, principalmente de su familia. Tercero, tomando en cuenta que la mayor parte del tiempo los estudiantes se la pasan en la universidad, algunas de las acciones sostenibles son difíciles de realizar, ya que esta no propicia el ambiente adecuado para llevar a cabo dichas acciones.

En el último aspecto, con base en la literatura consultada y a las respuestas de entrevistados, en cuanto a las acciones que consideran que debería realizar la universidad para un 

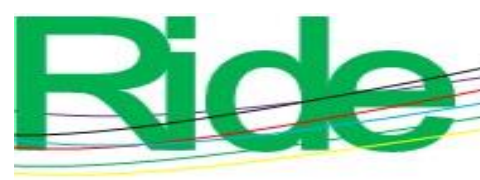

Revista Iberoamericana para la Investigación y el Desarrollo Educativo ISSN $2007-7467$

mayor cuidado del ambiente, se determinaron algunas acciones emergentes: se puede realizar una mayor difusión de la información en cuanto a los planes y acciones sobre el cuidado del ambiente que realiza la universidad, ya que la mayoría de los entrevistados desconocen si existe o no algún tipo de actividad relacionada con el cuidado del ambiente; podría contribuir a la mejora el aumentar la cantidad de botes de basura, puesto que existen estudiantes con hábitos de reciclaje, pero, argumentan, es difícil realizar dichas acciones cuando existe una cantidad insuficiente de contenedores de basura, así como la colocación de ceniceros, ya que se puede observar diariamente en las entradas de la universidad colillas de cigarros; es importante la realización de algún tipo de actividad relacionada con el cuidado del ambiente que incluya la participación de la comunidad universitaria, de todos los alumnos de todos los semestres de las licenciaturas, con la finalidad de que sean conscientes del impacto de sus acciones diarias y conozcan que esos actos son el camino hacia la sostenibilidad y, por lo tanto, saber en qué consiste el desarrollo sostenible.

Finalmente, se espera que estudio pueda contribuir a que futuras investigaciones pongan el énfasis no solo en las percepciones de los actores sociales respecto al tema de la sostenibilidad, sino también a las acciones que realizan. Así, valores y valoraciones se verán reflejados en los resultados de las mismas y en las propuestas.

\section{Agradecimientos}

Agradecemos la colaboración de los participantes en esta investigación, quienes amablemente firmaron un consentimiento informado para hacer posible la publicación de los resultados. 


\section{Referencias}

Caballero, M. (2011). Experiencia educativa dentro de un área natural protegida: centro de educación ambiental del Ajusco medio. Revista Transatlántica de educación, 9, 45-58. Recuperado de https://agua.org.mx/wpcontent/uploads/2012/02/trasatlanticadeeducacion_ambiental.pdf .

Conde, R., González, O. y Mendieta, E. (2006). Hacia una gestión sostenible del campus universitario. Laberinto, 93-94, 15-25. Recuperado de http://www.uam.mx/difusion/casadeltiempo/93_94_oct_nov_2006/casa_del_tiempo_num

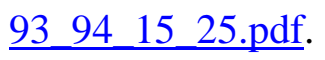

Dueñas, J. (2011). El papel de la educación ante el desarrollo sostenible. Revista Transatlántica de educación, 9, 15-20. Recuperado de https://agua.org.mx/wpcontent/uploads/2012/02/trasatlanticadeeducacion_ambiental.pdf.

García, S. (2011). La educación ambiental formal, base para la construcción de una nueva cultura del agua en los nuevos ciudadanos. Revista Transatlántica de educación, 9, 87-92. Recuperado de https://agua.org.mx/wpcontent/uploads/2012/02/trasatlanticadeeducacion_ambiental.pdf.

García Rendón, C. (2016). La construcción del ethos en el ámbito de la educación con una visión de sostenibilidad. En González, E y Vargas, H. (coords.), Educación ambiental transversal y transdisciplinaria: una visión decrecentista desde la ética, la cultura de paz. y el dialogo de saberes para una calidad de vida no-violenta. México: Torres Asociados.

García Rendón, C. y Panchi, V. (2015). Re-ligación, su contribución en la construcción del ethos. En Vargas, H. (coord.), Calidad de vida no violenta: saberes originarios, prácticas de paz y decrecimiento. México: Torres Asociados.

González, J. (1996). El ethos, destino del hombre. México: FCE.

Juárez, A, Juan, J. y Estrada, R. (2016). Gestión sostenible para el bienestar social universitario. El caso de una universidad pública del altiplano mexicano. Revista Caribeña de Ciencias Sociales. Recuperado de http://www.eumed.net/rev/caribe/2016/01/sostenibilidad.html.

Leff, E. (2011). La esperanza de un futuro sustentable: utopía de la educación ambiental. Revista Transatlántica de educación, 9, 93-103. Recuperado de https://agua.org.mx/wpcontent/uploads/2012/02/trasatlanticadeeducacion_ambiental.pdf. 


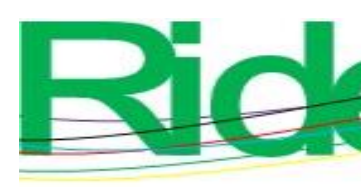

Revista Iberoamericana para la

Investigación y el Desarrollo Educativo

ISSN 2007 - 7467

Luengo, J. (2004). La educación como objeto de conocimiento. El concepto de educación. En del Pozo, M. (coord.), Teorías e instituciones contemporáneas de educación (pp. 30-47). Madrid, España: Biblioteca Nueva. Recuperado de http://www.ugr.es/ fjjrios/pce/media/1-EducacionConcepto.pdf.

Mendoza, R. (2016). Ética: formación y transformación humana. México: Torres Asociados.

Novo, M. (1996). La Educación Ambiental formal y no formal: dos sistemas complementario. Revista Iberoamericana de Educación, 11, 75-102. Recuperado de https://dialnet.unirioja.es/servlet/articulo? codigo=1020056.

Novo, M. (2003). El desarrollo sostenible: sus implicaciones en los procesos de cambio., Polis. Revista de la Universidad Bolivariana, 1(5). Recuperado de http://www.redalyc.org/articulo.oa?id=30500509.

Novo, M. (2005). Educación ambiental y educación no formal: dos realidades que se realimentan. Revista de Educación, (338), 145-165. Recuperado de http://www.revistaeducacion.mec.es/re338/re338_10.pdf.

Novo, M. (2009a). El desarrollo sostenible: su dimensión ambiental y educativa. Madrid, España: Universitas.

Novo, M. (2009b). La educación ambiental, una genuina educación para el desarrollo sostenible. Revista de Educación, (2009)195-217. Recuperado de http://www.revistaeducacion.mec.es/re2009/re2009_09.pdf.

Novo, M. (2010). Despacio, despacio. 20 razones para ir más lentos por la vida. Barcelona, España: Obelisco.

Novo, M. (2012). Educación ambiental. Bases éticas, conceptuales y metodológicas. Madrid, España: Universitas.

Parellada, R. (2008). Necesidades, capacidades y valores. Revista Papeles, (102), 77-87.

Recuperado de

http://www.fuhem.es/media/cdv/file/biblioteca/PDF\%20Papeles/102/Necesidades_capaci dades_valores.pdf

Peza, G. (2011). La educación ambiental en el espacio social de los estudiantes normalistas. El estudio de caso en las normales de Nuevo León. Avances de la investigación. Revista 

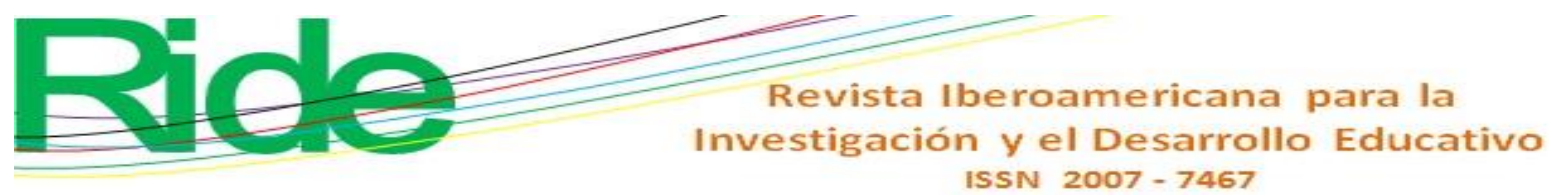

Transatlántica de educación, (9), 61-66. Recuperado de https://agua.org.mx/wpcontent/uploads/2012/02/trasatlanticadeeducacion_ambiental.pdf.

Sastre, A. (2011). Educar en la sostenibilidad. Agenda 21-Colegio San Francisco de Asís: "San Francisco hace un buen papel. Revista Transatlántica de educación, (9), 67-73. Recuperado de https://agua.org.mx/wpcontent/uploads/2012/02/trasatlanticadeeducacion_ambiental.pdf.

Organización de las Naciones Unidas para la Educación, la Ciencia y la Cultura [Unesco]. (2016). México: Grupo Ecológico Sierra Gorda, campeones de la tierra por el desarrollo sostenible. México: Unesco. Recuperado de http://www.unesco.org/new/es/office-inmontevideo/about-this-office/singleview/news/mexico_grupo_ecologico_sierra_gorda_campeones_de_la_tier/.

Organización de las Naciones Unidas [ONU]. (2015). Programa de las Naciones Unidas para el Medio Ambiente (PNUMA). ONU. Recuperado de http://e.exam10.com/doc/6765/index.html?page $=8$.

Yurén, M. (2001). Ethos profesional, eticidad y dispositivos de formación. En Hirsch, A. (coord.). Educación y valores. Tomo III. Ciudad de México, México: Gernika. 


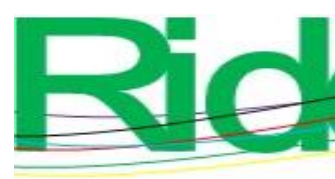

\begin{tabular}{|c|c|c|}
\hline Rol de Contribución & Definición (solo poner nombre del autor) & Grado de contribución \\
\hline Conceptualización & $\begin{array}{l}\text { Ideas; Formulación o evolución de objetivos y metas generales } \\
\text { de investigación. }\end{array}$ & Jenny y Cristina: Igual \\
\hline Metodología & Desarrollo o diseño de metodología; Creación de modelos. & $\begin{array}{l}\text { Cristina: Principal } \\
\text { Jenny: apoya }\end{array}$ \\
\hline Software & $\begin{array}{l}\text { Programación, desarrollo de software; Diseño de programas } \\
\text { informáticos; Implementación del código informático y } \\
\text { algoritmos de soporte; Pruebas de componentes de código } \\
\text { existentes. }\end{array}$ & No aplica \\
\hline Validación & $\begin{array}{l}\text { Verificación, ya sea como parte de la actividad o por separado, } \\
\text { de la replicación / reproducibilidad total de los resultados / } \\
\text { experimentos y otros productos de la investigación. }\end{array}$ & Jenny y Cristina: Igual \\
\hline Análisis Formal & $\begin{array}{l}\text { Aplicación de técnicas estadísticas, matemáticas, } \\
\text { computacionales y otras técnicas formales para analizar y } \\
\text { sintetizar los datos del estudio. }\end{array}$ & Jenny: Principal \\
\hline Investigación & $\begin{array}{l}\text { Llevar a cabo un proceso de estudio e investigación, } \\
\text { específicamente realizando los experimentos, o la recolección } \\
\text { de datos / evidencia. }\end{array}$ & Jenny: Principal \\
\hline Recursos & $\begin{array}{l}\text { Suministro de materiales de estudio, reactivos, materiales, } \\
\text { pacientes, muestras de laboratorio, animales, instrumentación, } \\
\text { recursos informáticos u otras herramientas de análisis. }\end{array}$ & Jenny y Cristina: Igual \\
\hline Curación de datos & $\begin{array}{l}\text { Actividades de gestión (producir metadatos), depurar } \\
\text { información y mantener datos de investigación (incluyendo } \\
\text { código de software, donde sea necesario para interpretar los } \\
\text { datos en sí) para uso inicial y posterior reutilización. }\end{array}$ & Jenny y Cristina: Igual \\
\hline $\begin{array}{l}\text { Escritura - Preparación del } \\
\text { borrador original }\end{array}$ & $\begin{array}{l}\text { Creación y / o presentación de la obra publicada, escribiendo } \\
\text { específicamente el borrador inicial. }\end{array}$ & Cristina: principal \\
\hline Escritura - Revisión y edición & $\begin{array}{l}\text { Preparación, creación y / o presentación del trabajo publicado } \\
\text { por parte del grupo de investigación original, específicamente } \\
\text { revisión crítica, comentario o revisión, incluidas etapas previas } \\
\text { o posteriores a la publicación. }\end{array}$ & Cristina: Principal \\
\hline Visualización & $\begin{array}{l}\text { Preparación, creación y / o presentación del trabajo publicado, } \\
\text { específicamente visualización / presentación de datos. }\end{array}$ & Cristina: Principal \\
\hline
\end{tabular}




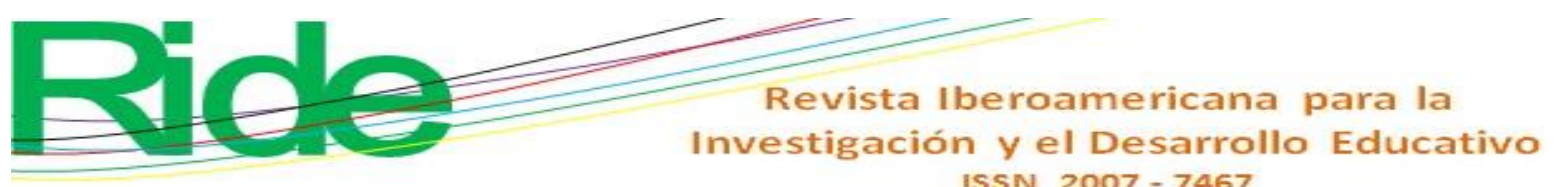

\begin{tabular}{|l|l|l|}
\hline & & Jenny: Apoya \\
\hline Supervisión & $\begin{array}{l}\text { Responsabilidad de supervisión y liderazgo en la planificación y } \\
\text { ejecución de actividades de investigación. }\end{array}$ & Cristina: Principal \\
\hline Administración de Proyectos & $\begin{array}{l}\text { Responsable de la gestión y coordinación de la planificación y } \\
\text { ejecución de las actividades de investigación. }\end{array}$ & Cristina: Principal \\
\hline Adquisición de fondos & $\begin{array}{l}\text { Adquisición del apoyo financiero para el proyecto que conduce } \\
\text { a esta publicación. }\end{array}$ & $\begin{array}{l}\text { Proyecto sin } \\
\text { financiamiento } \\
\text { institucional. } \\
\text { Cristina: Principal }\end{array}$ \\
\hline
\end{tabular}

